Research Article

\title{
Filter Realization of the Time-Domain Average Denoising Method for a Mechanical Signal
}

\author{
Wuwei Feng $\left(\mathbb{D},{ }^{1,2}\right.$ Cuizhu Wang $\mathbb{D},{ }^{1}$ Xin Chen $\mathbb{D}^{1},{ }^{1}$ Yuzhou Shi $\mathbb{D},{ }^{1}$ Meng Jiang $\left(\mathbb{D},{ }^{1}\right.$ \\ and Di Wang $\mathbb{1}^{1}$ \\ ${ }^{1}$ School of Naval Architecture and Mechanical-Electrical Engineering, Zhejiang Ocean University, Zhoushan 316022, China \\ ${ }^{2}$ Dong Hai Institute of Science and Technology, Zhejiang Ocean University, Zhoushan 316022, China \\ Correspondence should be addressed to Cuizhu Wang; 1030779284@qq.com
}

Received 5 November 2020; Revised 11 April 2021; Accepted 15 April 2021; Published 27 April 2021

Academic Editor: Radoslaw Zimroz

Copyright (C) 2021 Wuwei Feng et al. This is an open access article distributed under the Creative Commons Attribution License, which permits unrestricted use, distribution, and reproduction in any medium, provided the original work is properly cited.

Time-domain averaging (TDA) is an effective signal processing technique in fault diagnosis that can extract the periodic components of interest from signals mixed with noise interference while suppressing other irrelevant periodic signals. However, there are two obvious shortcomings to TDA: first, the acquisition of keyphasor signals is often restricted by the application environment and conditions. Even if the signal is obtained by TDA, owing to the existence of periodic truncation errors, satisfactory results cannot be obtained. Second, due to the velocity fluctuation, the actual mechanical signal is easy to produce a large error in TDA stacking. To solve the above challenges, first, based on the disadvantage of using traditional resampling to solve the TDA synchronization problem, this paper proposes a new method of subsection resampling, which improves the analysis effect of the traditional TDA. Second, to further expand the range of the practical applications, according to the amplitudefrequency map of TDA, a method for realizing TDA function by using the FIR multiband filter is proposed. This approach effectively avoids the requirement of traditional methods to collect the keyphasor signals and broadens the application in practical engineering. Finally, the improved TDA method is compared with the filter implementation technology, and their respective application conditions are given.

\section{Introduction}

Vibration signal processing has been an effective means to monitor mechanical equipment for many years [1]. However, mechanical vibration signals are usually masked by significant background noise, which leads to many research and development of denoising methods. In the domain of signal denoising, the existing technologies include Fourier transform, short-term Fourier transform, wavelet analysis, empirical mode of decomposition, and so on. Although Fourier transform is used primarily for decomposition, it is not suitable for nonstationary signal decomposition. In order to overcome this defect, an improved short-term Fourier transform method (STFT) is utilized, but it sacrifices the frequency resolution to capture the transient behavior of the signal. The resolution problem of STFT is solved by wavelet transform based on multiresolution analysis. While wavelet transform is a nonadaptive method, which cannot eliminate the high-frequency noise in the signal and is poor at dealing with nonlinear data. EMD technology, which can eliminate high-frequency noise from nonlinear and nonstationary signals (such as ECG), is an adaptive method. Ghosh [2] analyzed that EMD is superior to the other methods discussed above in dealing with nonlinear nonstationary signals. Some scholars combine these methods for research, such as Wang et al. [3] proposed an improved threshold wavelet denoising algorithm based on EMD. Some scholars have proposed some new methods, such as Premanode et al. [4] using the averaging intrinsic mode function to denoise the nonlinear and nonstationary time series data. Due to the complexity of the algorithm and the heavy workload of calculation, it needs long-term exploration to extend the above methods to practical application.

Time-domain averaging (TDA) or time synchronization averaging (TSA) is widely used in practice with its simple 
algorithm. It mainly uses the principles of random statistics to stack and average the original signal for many times to eliminate or reduce the noise influence from other sources, to further enhance the signal components of interest [5-8]. It is often used for early fault detection of gears to eliminate irrelevant signal noise and extract weak fault features [9-13]. Traditional TDA has perfect processing effect for stationary signals, but there still exist some shortcomings for nonstationary signals. Strictly speaking, most mechanical vibration signals are nonstationary, for example, signal speed fluctuation caused by benign operational and environmental variations. The velocity fluctuation will make the superposition of TDA difficult or produce large error, so it is urgent to study the improved TDA algorithm for nonstationary signals. Many scholars have done a lot of research on this problem. Rahman et al. [14] proposed the impact-synchronous time averaging technique to solve the problem that the time averaging method cannot process asynchronous signals. Ahamed et al. [15] proposed a multiple-pulse individually rescaled time synchronous averaging technique combined with the traditional TDA and developed a robust method for gear fault detection under fluctuating load and speed conditions. In 2011, Cross et al. [16] introduced the concept of cointegration to deal with the nonstationary time series and remove the environment and operation trend from the original data. The theory of cointegration arose in the field of econometrics, which is mainly used to analyze and understand the common trends in economic and financial time series $[17,18]$. It can combine multiple nonstationary time series to produce stationary residuals $[19,20]$. TDA resampling technology is also able to process nonstationary signals to ensure good linear superposition [21, 22]. For example, Zhao and Guo [23] used digital resampling technology to solve this problem. These methods have positive effects in processing nonstationary signals.

In summary, the research studies in this domain have enhanced the analysis effect of TDA from different aspects, but most of the research studies only focus on improving the traditional methods. For the problem that it is very difficult to obtain the keyphasor signal in complex and harsh environment, further research is still required. In order to successfully apply the TDA in complex environment, this paper first studies the traditional TDA and points out the shortcoming of using resampling to correct the synchronization problem of TDA. On the basis of the traditional TDA, we propose a subsection resampling method to improve this shortcoming. The simulation and experimental results shows that the segmented resampling can effectively improve the analysis accuracy and has well application value. Secondly, to solve the difficulties of acquisition keyphasor signal in complex environment, a filter implementation technique is proposed to realize the TDA function without the keyphasor signal. The simulation and experimental results show the feasibility and effectiveness of the filter implementation technology. Finally, the improved TDA is compared with the method using the filter implementation technology, and their application conditions are given.

\section{Improvement of the Traditional TDA Denoising Method}

TDA not only needs to pick up the signal to be analyzed but also needs to acquire the timing pulse of the rotation axis to lock the starting point of each signal segment. Since the signal is digital on average, the same number of points is required for each segment of data. Because the sampling frequency of $A / D$ converter is constant once set and the timing pulse frequency and the period of the signal to be analyzed also change at any time due to the change of mechanical speed even if the small change, it is impossible to guarantee the equal number of data of each segments by conventional sampling. The method to solve this problem is to use frequency tracking technology so that the actual sampling frequency can track the rotation frequency in real time and equal to the integer multiple of it.

The key of TDA is signal synchronization. If the signal is asynchronous, the useful components of the signal after the TDA processing will be attenuated, leading to the preset purpose not being achieved.

At present, frequency tracking technology is generally used to solve the problem of signal asynchronization caused by various factors $[8,24]$. One method uses hardware to calibrate and collect the fluctuation signals during data acquisition and another method processes the asynchronous signals after data acquisition via software using data resampling technology to realize frequency tracking. To avoid frequent mixing, the final actual sampling frequency should be more than twice the bandwidth of the signal. In this paper, the method of software is adopted, that is the resampling method.

Specific approach of the traditional resampling:

The analyzed signal $x(t)$ and time-scale pulse $e(t)$ are input into the two-channel A/D converter to obtain the long-series $x_{n}$ and $e_{n} \cdot x_{n}$ is divided into several data segments $x_{n}^{p}(p=0,1,2,3, \cdots, P)$ based on $e_{n}$. Now, each data segment is not equal in number of points. This is the first sampling. Then, the second sampling, namely, the resampling process, is carried out. Under the premise of satisfying the sampling theorem, the sampling signal $x_{n}$ is restored to the original signal $x(t)$, as follows:

$$
x(t)=\sum_{n=-\infty}^{\infty} x_{n} \frac{\sin (\pi(t-n \Delta) / \Delta)}{\pi(t-n \Delta) / \Delta} .
$$

Assume that the number of points of a data segment sampled for the first time is $M$ and the number of points of unified sampling for each data segment is $N$. Resampling of the above recovery signal $x(t)$ is carried out adaptively with the resampling interval $\delta=M / N$, and the result is given by formula (2). Thus, data segments $P$ with the exact same number of points are obtained, and the number of sampling points for each segment is $N$ :

$$
x_{r}=\sum_{n=-\infty}^{\infty} x_{n} \frac{\sin (\pi(r \delta-n \Delta) / \Delta)}{\pi(r \delta-n \Delta) / \Delta} \quad r=0,1,2, \ldots, N,
$$

where 


$$
\delta=M / N \text { is the resampling interval. }
$$

According to the uniform sampling number $N$ and the different first sampling number $M$ of each segment, each data segment is resampled with an adaptive sampling interval $\delta$, so as to achieve frequency tracking and phase synchronization.

The sampling interval $\delta$ used in traditional resampling is carried out under the default condition that the signal velocity is constant within a truncation period, that is, the sampling interval is the same within each truncation period. However, due to the existence of velocity fluctuations, the signal data points within a segment will not be at the same velocity, so that the collected data segment is out of sync. Therefore, a segmented resampling method is proposed in this paper.

\subsection{Software Implementation Technology of Frequency} Tracking. Software implementation technology of frequency tracking mainly refers to the subsequent processing of the collected asynchronous signals, which mainly uses a programming language to implement certain algorithms to synchronize the collected signals, and frequency tracking is realized by the data resampling technique.

The sampling interval adopted by traditional data resampling technology is carried out under the default constant speed condition within a section of the data signal, that is, the sampling interval is the same for the data within a section, and its procedure is shown in Figure 1. However, due to the existence of speed fluctuation, the speeds of the signal data points within a section are unequal, leading the collected data segment to be asynchronous. Therefore, a segmented resampling technique is adopted in this paper to improve this situation. The procedure of this method is shown in Figure 2:

The only difference between the improved segmented resampling technique and the traditional resampling technique is that the sampling interval of the segmented resampling of the whole truncated data is determined according to the situation. The specific method of segmented resampling is as follows: after the long signal is truncated, each segment signal to be superposed is divided into four parts at phases of $0, \pi / 2, \pi$, and $3 \pi / 2$. In the case where there is no velocity fluctuation by default but there is, when the data collected by each part is too dense due to the velocity change, the segmented resampling method adopts a lower sampling rate to resample to ensure that the final points of each data segment are equal [25]. In the same way, when the collected data points are too sparse, resampling correction is carried out with a higher sampling rate. It should be noted that resampling each small segment separately is not done by cutting the truncated signal into four segments but by adopting stretch sampling or compression sampling, according to the situation, for the data points within the four segments. The flowchart of the segmented resampling technique is shown in Figure 3:

When the rotation frequency is not available, which means the exact period of the periodic component of the signal is not clear, the period can be determined by obtaining the maximum value of the autocorrelation coefficient [26].

Both the cointegration method and the multiple regression method study the correlation between variables. Cointegration method is mostly used to study the relationship between economic variables [27-29], and multiple regression analysis is used to study the linear or nonlinear relationship between multiple variables, which is applied in various fields [30]. In the field of machinery, it is mostly used in the state trend prediction analysis of mechanical equipment [31, 32]. In the segmented resampling, the signal of each segment also has cointegration relationship, and the fluctuation trend of each segment is basically the same. However, the research purpose of this paper is to extract the periodic component from the signals, and less attention is paid to the cointegration relationship of the signal, so the method proposed in this paper is simpler and more effective.

2.2. Numerical Simulation. A composite signal $s(t)$ is constructed to verify the validity of the segmented resampling method and is mainly composed of three parts, including the power frequency signal of the rotating machinery $f(t)$, the simulated impact signal $g(t)$, and the white noise signal $x(t)$. The power frequency signal $f(t)$ is the mechanical rotation signal under the ideal state. For example, in this paper, the rotation signal waveform in the ideal state is sinusoidal when the small rotor test bed is under the condition of misalignment. Expression of the composite signal is $s(t)=f(t)+g(t)+x(t)$. In the synthetic simulation signal, the impact is basically submerged in the noise signal. To simulate the fluctuation situation, the number of data points collected in each phase of the power frequency signal $f(t)$ is changed when constructing the signal. The respective signals are illustrated in Figure 4.

The composite signal was processed according to Figure 3. Figure 5 shows the figures of the TDA results of the traditional resampling method, the segmented resampling proposed in this paper, and the ideal situation of synchronous stack averaging. Comparing Figures 5(a)-5(c), the results demonstrate that segmented resampling has the same effect as the ideal synchronous averaging method, and the impact signal is highlighted. The traditional resampling method weakens the impact signals, as shown in Figure 5(a). Therefore, the segmented resampling method proposed in this paper effectively improves the analysis accuracy of TDA.

2.3. Experimental Validation. In this section, the improved TDA, the segmented resampling, is applied to the actual vibration signal of the bearing rotation collected in the small rotor test bed under the condition of misalignment. The BK3000XL type eddy current sensor with a probe of $\varphi 5$ is mounted on the shaft to collect the radial displacement signal of the shaft. The picture of the test rig is shown in Figure 6. This method requires accurate mark points of the phase.

The parameters of the small rotor test bed are set as follows: the sampling frequency is $1600 \mathrm{H}_{Z}$, the rotation speed is $3000 \mathrm{rpm}$, the data acquisition card used is Linghua 


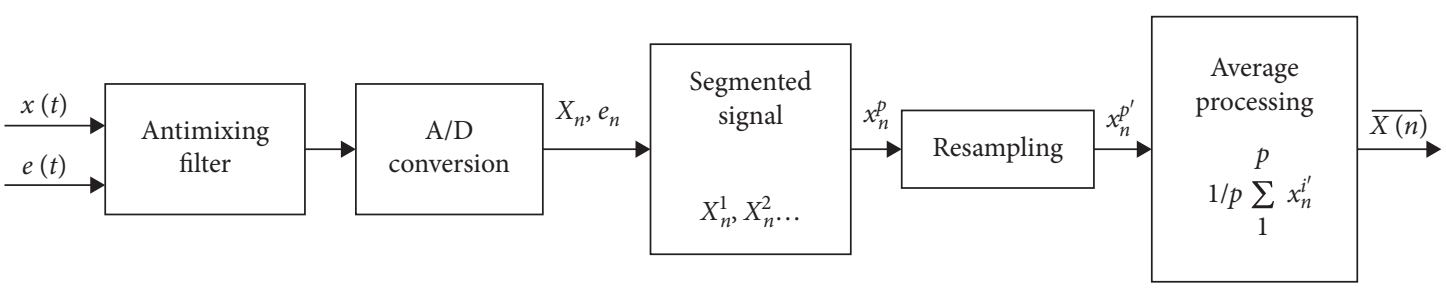

Figure 1: Traditional process.

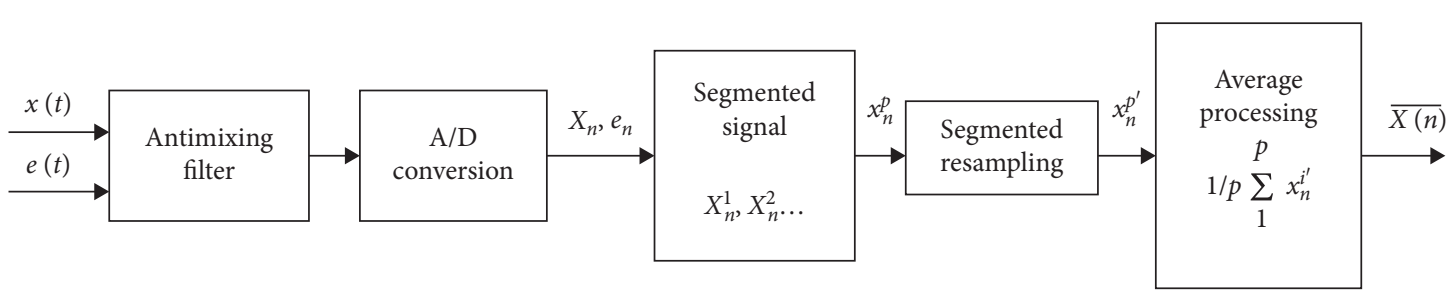

FIGURE 2: Improved process.

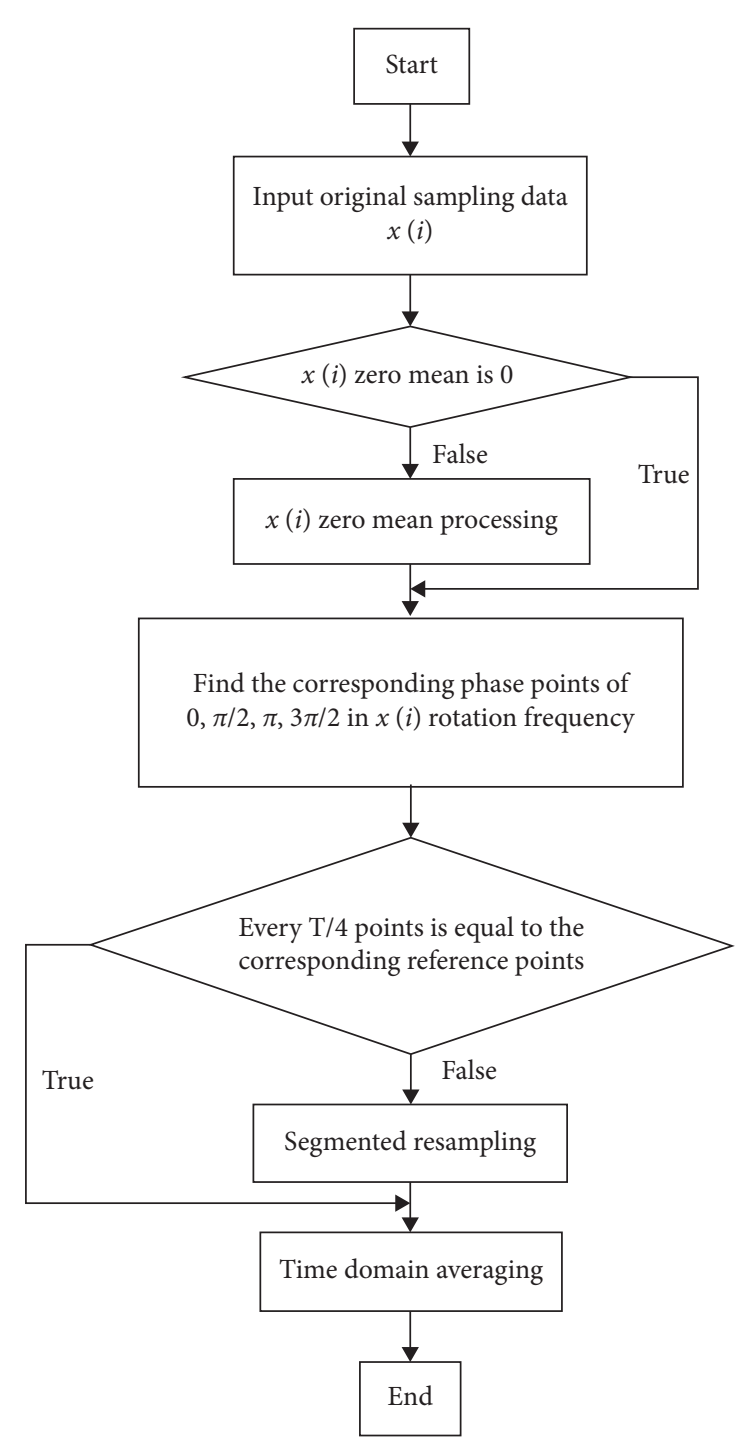

Figure 3: The implementation flow chart. $\mathrm{T}$ is the rotation period of rotary machinery, which is generally used as the truncation period.
DAQ2213, the A/D conversion digit is 12, and the keyphasor signal trigger acquisition mode is adopted. The fault signal in this test is obtained under the condition of a mechanical turntable collision and wear fault. Due to external and structural influences, the velocity fluctuation is approximately $8 \%$ when the system is stable. The partial amplification figure of the collected signals is shown in Figure 7, where the signal with the large amplitude is the keyphasor signal of the rotating machinery and the other is the vibration signal of the shaft.

The collected signals are analyzed and processed by traditional resampling and the segmented resampling proposed in this paper, and the results are presented in Figures 8 and 9. From Figure 8, it can be seen that there is still a phase difference in the signal with the velocity fluctuation processed by the traditional resampling method, the amplitude of the analog fault signal superimposed and averaged tends to decay, and the fault characteristics are reduced or even submerged. From Figure 9, it can be seen that the phase difference of the superimposed data segment is obviously improved, as shown in Figure 9(a), and the synchronicity after processing is well enhanced, as shown in Figure 9(b). The superimposed signal can effectively highlight the mechanical rubbing fault, achieving a good expected effect.

\section{Filter Implementation Technology of TDA}

3.1. Principle. Obtained from the TDA formula, the amplitude-frequency response is shown in equation (4), and the phase is shown in equation (5):

$$
\begin{aligned}
|H(f)| & =\frac{1}{N}\left|\frac{\sin \left(\pi N f / f_{0}\right)}{\sin \left(\pi f / f_{0}\right)}\right|, \\
\varphi(f) & =\arg H(f)=\pi(N-1) \frac{f}{f_{0}} .
\end{aligned}
$$

Different segment numbers $N(N=8, N=16$, and fundamental frequency $f_{0}=50 H_{Z}$ ) are applied in equations 


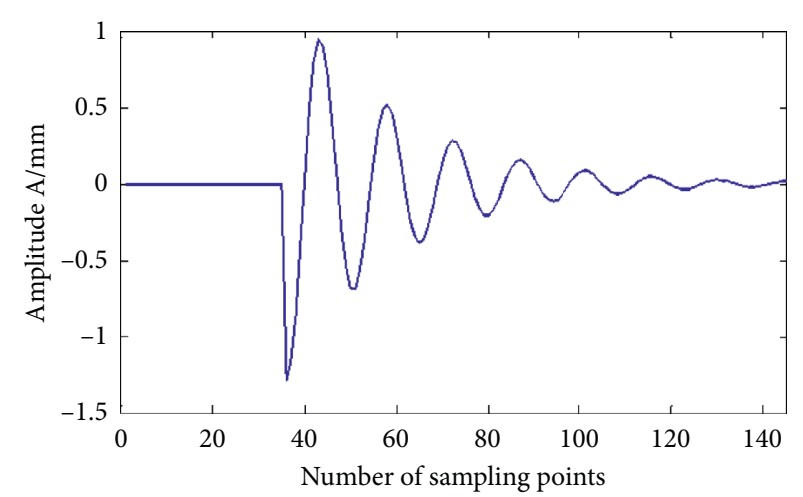

(a)

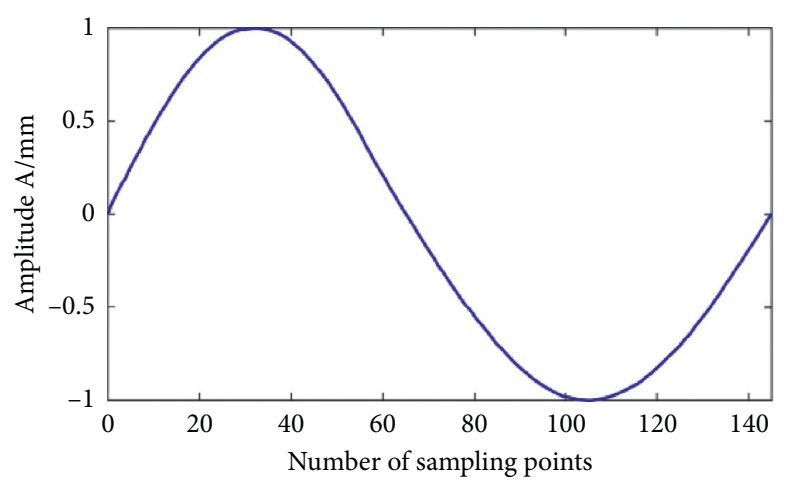

(c)

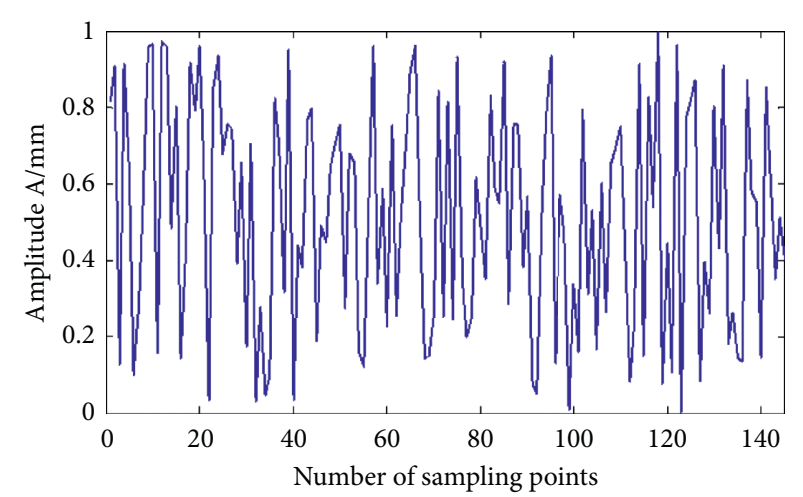

(b)

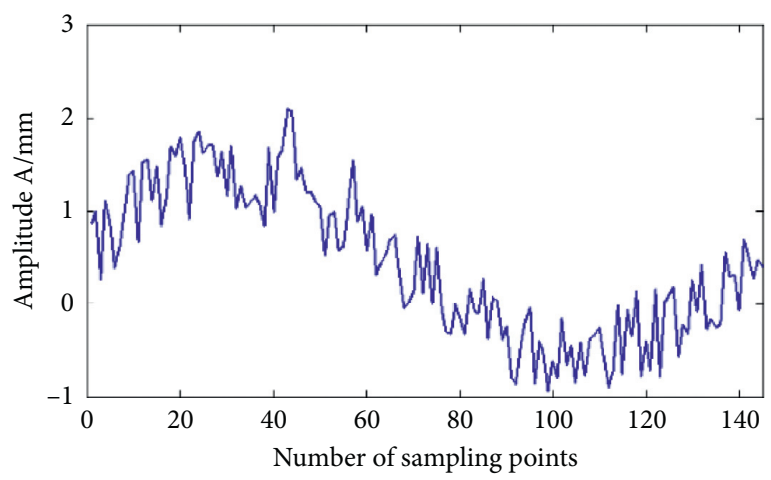

(d)

FIgURE 4: Simulation signal. (a) The simulated impact signal $g(t)$, (b) the white noise signal $x(t)$, (c) the power frequency signal $f(t)$, and (d) composite signal $s(t)$.

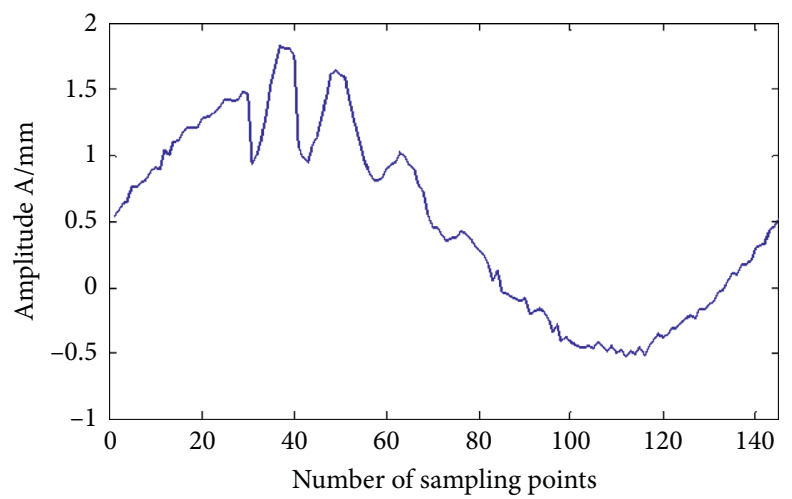

(a)

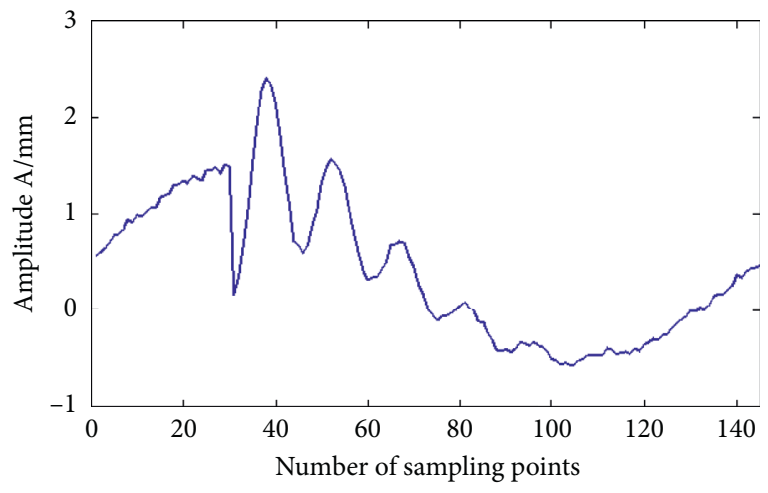

(b)

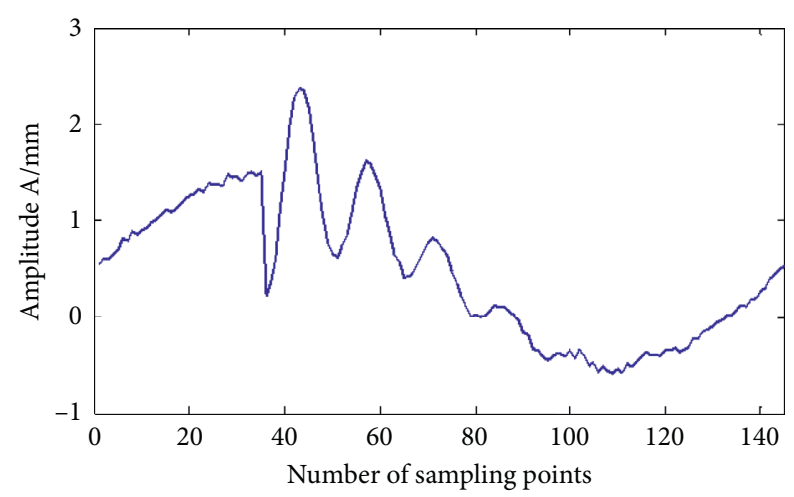

(c)

FIGURE 5: Comparison of treatment results of different methods. (a) Traditional processing results, (b) improved treatment results, and (c) ideal synchronization results. 


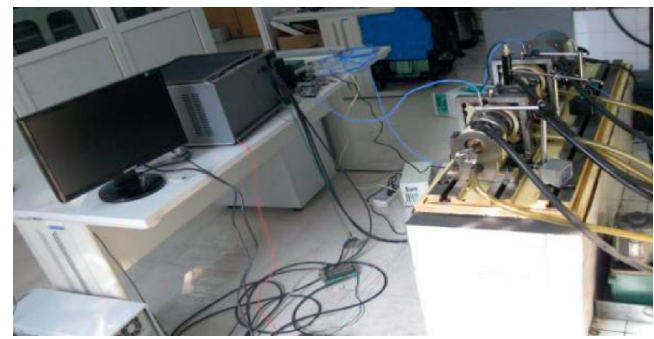

Figure 6: The picture of the test rig.

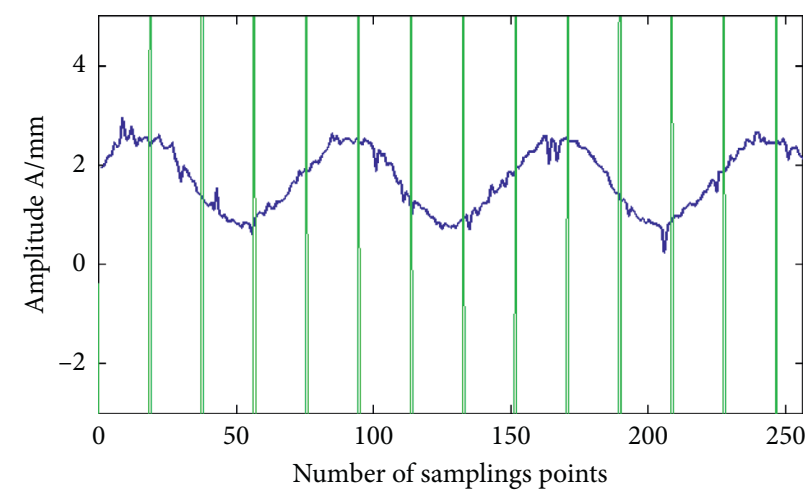

FIgURE 7: Time-domain waveform of the acquisition signal.

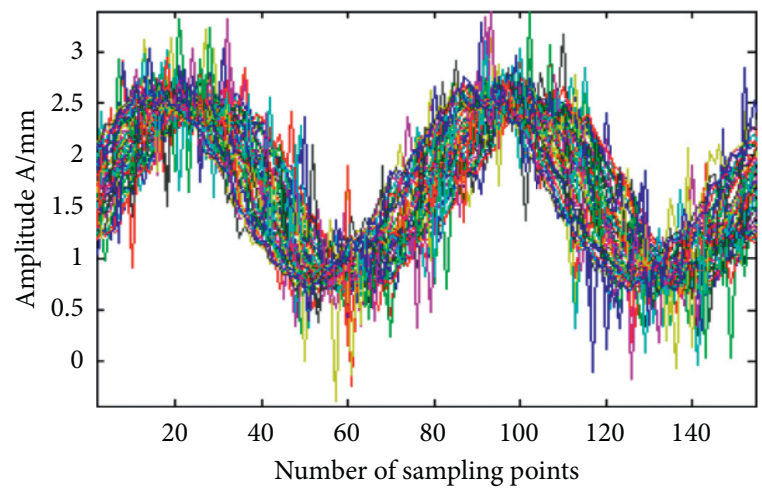

(a)

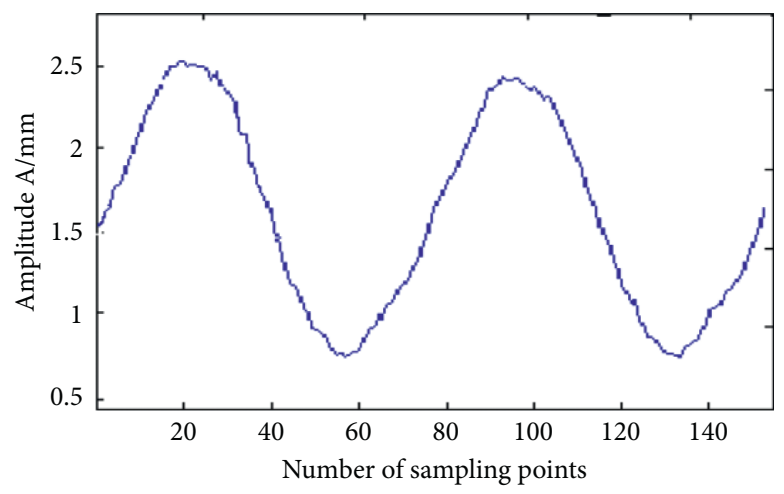

(b)

FIgURe 8: Traditional TDA processing results. (a) Synchronous correction data segment before improvement and (b) stacking average result.

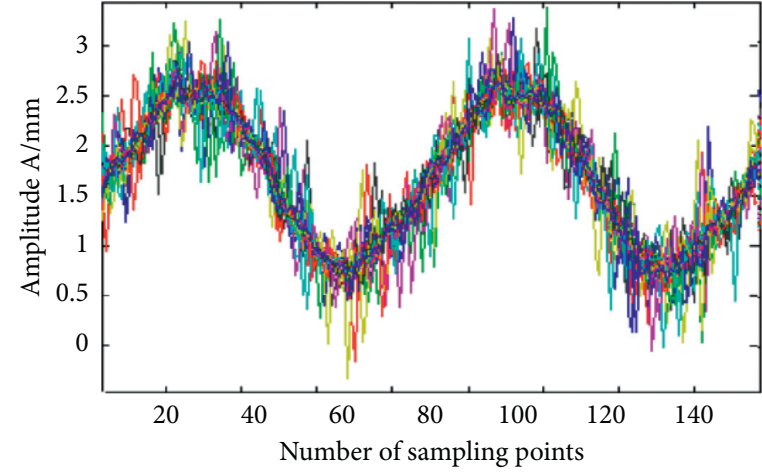

(a)

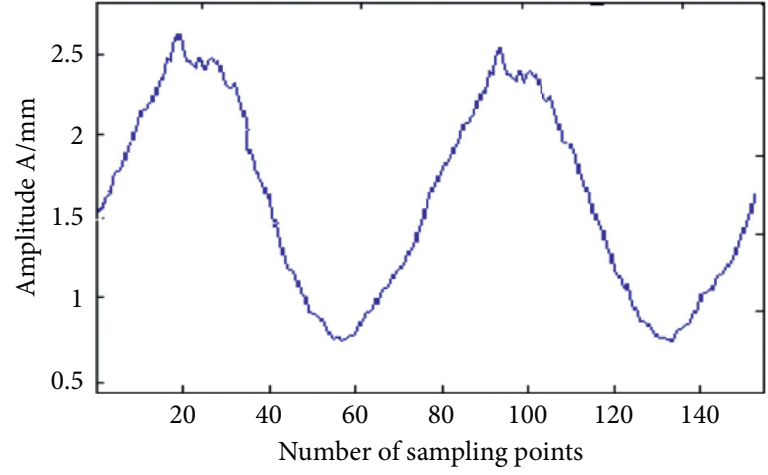

(b)

FigURE 9: Improved TDA processing results. (a) Improved synchronous correction data segment and (b) stacking average result. 


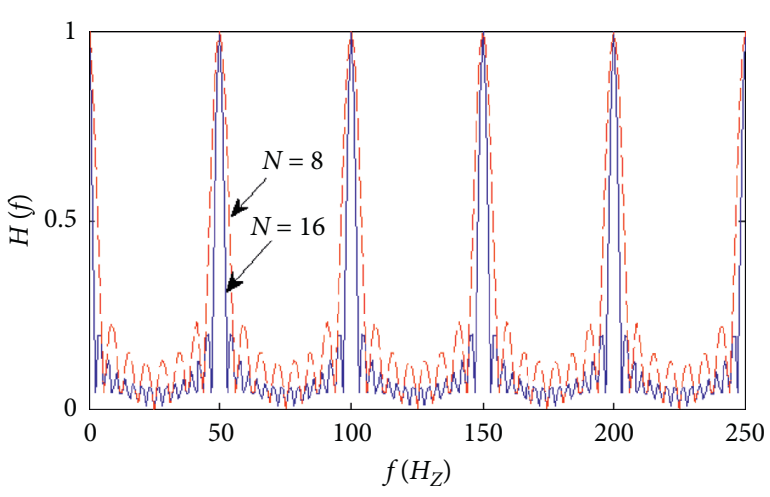

FIgURE 10: Amplitude-frequency response of TDA.

(4) and (5), and the obtained amplitude-frequency diagram is shown in Figure 10:

From Figure 10, the response curve is composed of a series of equal interval distributed bandpass comb curves and side lobes [33]. With the increase in the number of superimposed segments $N$, the comb curve with equal intervals becomes sharper (as seen from the solid line and dotted line in Figure 10), while the side lobe is more obviously suppressed. This is the reason the time-domain synchronous average can effectively extract the periodic signals related to the rotation frequency and has a strong suppression ability for noise and noncorrelated signal components. Based on the characteristics of the amplitude response graph of TDA, the process of extracting periodic components can be regarded as the process of filtering the signals.

In fact, there are many kinds of signal denoising methods, such as wavelet analysis and empirical mode decomposition. Wavelet analysis (WV) [34-36], especially the harmonic wavelet, has obvious advantages in signal processing. In theory, the harmonic wavelet is a perfect bandpass filter, which can be inferred from the "box spectrum" of the harmonic wavelet. Multiband filtering can be determined at random according to the fundamental frequency. However, wavelet filtering applies a kind of frequency division processing process. Therefore, the TDA function cannot be achieved by wavelet filtering. Other signal processing methods, such as empirical mode decomposition (EMD) [37-39], focus on processing nonstationary signals and ignore the phase information, so they are also not suitable for this method. In summary, wavelet analysis and empirical mode decomposition have achieved good results in some fields, but they are powerless for the realization of the TDA function. They use a process to extract certain frequency signals from the original signal rather than eliminating the irrelevant signals. Only the effect of multiband filtering is consistent with the target of TDA. Therefore, the next step is to study the realization of TDA by multiband filtering.

3.2. Selection of Filter. The selection of the filter is critical for the realization of the TDA function. There are various kinds of filters, among which digital filters are mainly classified into FIR and IIR filters. The biggest advantage of FIR filter is that it has a linear phase and stable system structures and can realize zero phase shift filtering. To obtain a better filtering characteristic curve, the filtering order is usually selected to be larger. However, the disadvantages of FIR filter are the relatively large amount of computation required and the poor real-time performance of signal processing. The greatest advantage of IIR filter is that it can obtain accurate passband and stopband edge frequencies and effective passband and stopband attenuation. However, the disadvantage is that the system has a stability problem, and the linear phase relationship cannot be guaranteed for the processed signals. Since the TDA highly requires phase, to ensure that there is no phase shift between the fundamental frequency of a specific periodic component and its frequency doubling, a zero phase shift bandpass filter is designed from the FIR digital filtering technology to realize the TDA function [40].

\subsection{Filter Implementation of TDA}

3.3.1. Construction of the Multiband Filter. A finite length sequence is sought as the unit impulse response $h(n)$ of the system to make the system's frequency response $H\left(e^{j \omega}\right)$ approximate the expected frequency characteristic $H_{d}\left(e^{j \omega}\right)$. The window method was adopted to design the multiband filter. Its design idea is shown in Figure 11. The specific design of the multiband filter is

From Figure 11, the specific design [41] of multiband filter is:

(1) Determining the ideal frequency characteristic of the multiband filter $H_{d}(f)$

The multiband filter needs to retain the frequencies around the fundamental frequency and its octave, i.e. That is $f_{0}$ and $k f_{0}$ are the center frequencies, and the two sides $\pm P$ are respectively passbands, which need to be selected. Therefore, its ideal frequency characteristic is:

$H_{d}(f)= \begin{cases}1, & k f_{0} \pm p \quad k=0, \pm 1, \pm 2, \ldots \ldots, \\ 0, & \text { others. }\end{cases}$

(2) Unit sampling response:

$$
h_{d}(n)=F^{-1}\left[H_{d}(f)\right]=\frac{\sin (P n T)}{2 n \pi} \sum_{k=1}^{K} \cos \left(k f_{0} n T\right),
$$

where the symbol $K$ indicates the number of passbands of the multiband filter, $T$ is the sampling period and $f_{0}$ is the fundamental frequency.

(3) Selection of window function $\omega(n)$ :

Under the condition that the stopband attenuation meets the requirements, the window function with the narrow main lobe and small side lobe peak should be selected as far as possible. Compared with 


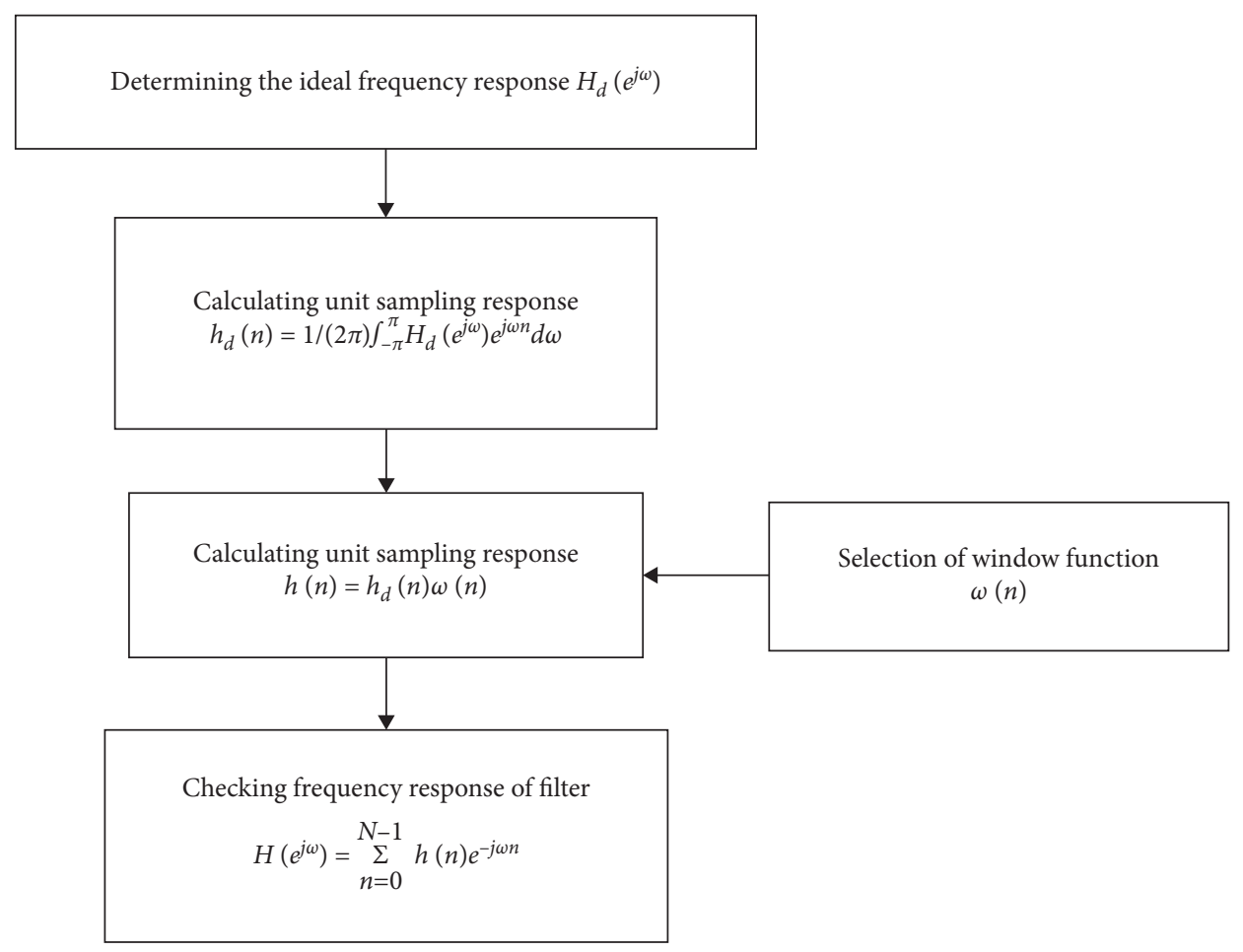

FIgUre 11: The design idea of multiband filter.

rectangular window, Hamming window and Hanning window are commonly used and have slightly wider main lobes, but smaller side lobes and larger attenuation speeds [41, 42]. Here, the Hanning window is selected as the window function.

$\omega(n)=\frac{1}{2}\left(1+\cos \left(\frac{2 \pi n}{N}\right)\right), \quad n=-\frac{N}{2}, \ldots 0, \ldots n=\frac{N}{2}$.
(4) Calculating the unit impact response:

$$
h(n)=h_{d}(n) \omega(n) .
$$

Namely:

$$
h(n)=\sum_{k=1}^{K} \frac{\sin \left(2 \pi\left(f_{k}+P\right) n T-\sin \left(2 \pi\left(f_{k}-P\right) n T\right)\right)}{\pi n} *\left(1+\cos \left(\frac{\pi n}{N}\right)\right),
$$

where the symbol $P$ represents the half bandwidth of the channel and $N$ is the filter order. After the above four steps, under ideal conditions, the unit impulse response $h(n)$ and its frequency characteristics of the constructed multiband filter are shown in Figure 12 (where $N=400, K=7$ ), and the selection of $K$ should be $K$ times $f_{0}$ less than $f_{s} / 2$.

\subsubsection{Determination of Fundamental Frequency and Octave} Frequency. One of the key technologies involved in the construction of multiband filter is the determination of the fundamental frequency and its octave frequency. The error of fundamental frequency $f_{0}$ will directly lead to the cumulative dislocation of the central frequency of each passband, which is illustrated by equation (6). The reason why the fundamental frequency cannot be accurately determined is usually due to spectrum leakage of the common FFT [43]. A computer can only calculate a finite number of samples, and various spectrum analysis methods are only carried out in a finite interval, which inevitably leads to the energy leakage caused by the truncation of the time domain and the fence effect caused by the limited number of analysis points. As a result, the spectrum peak of the frequency spectrum is reduced, that is, the amplitude, phase and frequency of the discrete spectrum may produce large errors, affecting the accuracy of the analysis. To solve this problem, frequency domain interpolation is adopted in this paper.

For discrete signal $x_{n}$, FFT is used to obtain discrete spectrum $X_{n}$, and then amplitude spectrum $\left|X_{n}\right|$ is gained. If the maximum spectral line near the fundamental frequency in the amplitude spectrum is $\left|X_{\max }\right|$, its corresponding 


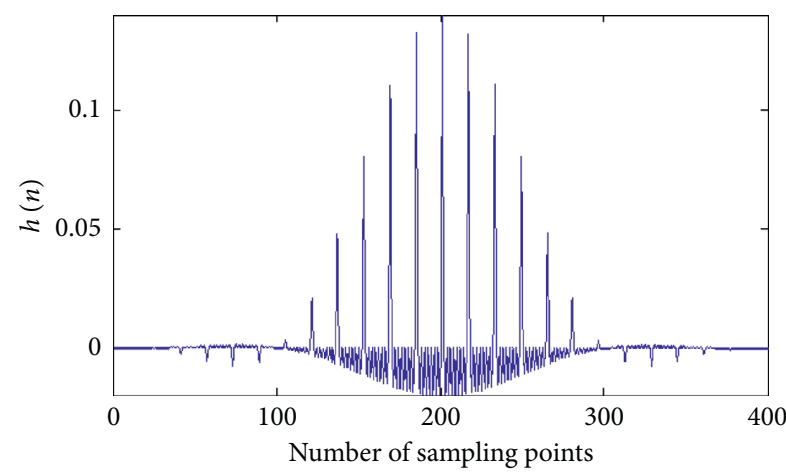

(a)

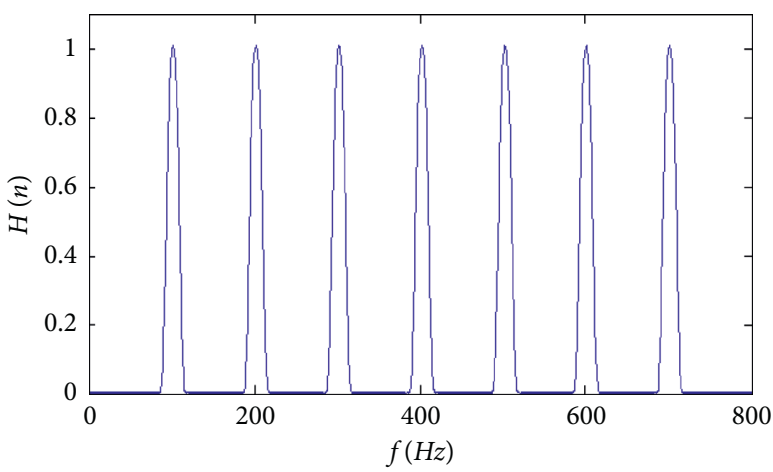

(b)

Figure 12: Response curve of multiband filter. (a) Impact response and (b) frequency characteristic.

frequency is approximation $\tilde{f}_{0}$ of the fundamental frequency, as illustrated in Figure 13. Generally, $\widetilde{f}_{0}$ is not equal to the real value $f_{0}$ and exhibits a certain deviation. Therefore, frequency interpolation is used to correct it to obtain the accurate value $f_{0}$.

Set $\left|X_{\text {minor }}\right|$ as the largest spectral line on both sides of $\left|X_{\max }\right|$, and the correction frequency is determined by equation (11):

$$
f_{0}=\tilde{f}_{0}+\delta * \Delta f
$$

where the symbol $\Delta f$ is the frequency interval and $\delta$ is the frequency correction factor.

$$
\delta= \pm \frac{\left|X_{\operatorname{minor}}\right|}{\left|X_{\text {minor }}\right|+\left|X_{\max }\right|} .
$$

Equation (12) takes the negative value when $\left|X_{\text {minor }}\right|$ is on the left of $\left|X_{\max }\right|$; otherwise, it takes the positive value.

After the fundamental frequency of the period is determined, the frequency multiplication is obtained by equation (13).

$$
f_{k}=k \cdot f_{0}, \quad k=1,2, \ldots, K,
$$

where the symbol $K$ is the number of octaves.

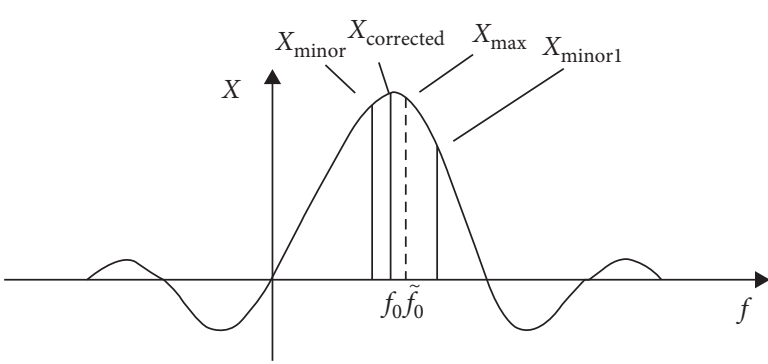

FIGURE 13: Interpolation correction diagram.

3.4. Numerical Simulation. The damage signal of the gear tooth surface is simulated in this paper. When the gear tooth surface is evenly worn, which causes the tooth gap to increase, or even there is cracking, pitting, peeling, and other damage on the tooth surface, the impact vibration will be generated, and the gear will be excited to vibrate according to its natural frequency. In the frequency component, the second and third harmonics or the frequency division component of $1 / 2,1 / 3$, etc., of the meshing frequency are generated. Suppose the analog discrete signal is composed of a periodic signal and random white noise, as follows:

$$
\begin{aligned}
x(n)= & \sin \left(2 \pi f_{m} n T\right)+0.2 \sin \left(4 \pi f_{m} n T\right)+0.33 \sin \left(6 \pi f_{m} n T\right)+ \\
& 0.1 \sin \left(8 \pi f_{m} n T\right)+\sin \left(2 \pi f_{1} n T\right)+\sin \left(2 \pi f_{2} n T\right)+ \\
& 0.4 \sin \left(2 \pi f_{3} n T\right)+r(n),
\end{aligned}
$$

where the signal is composed of the fundamental frequency $f_{0}=f_{m}=50 H_{Z}$ (meshing frequency), its frequency multiplication is $2 f_{m}, 3 f_{m}$, and $4 f_{m}$, the sinusoidal signals with frequency $f_{1}, f_{2}$, and $f_{3}$ and white noise $r(n)$. As the frequency of the interference signal, $f_{1}, f_{2}$, and $f_{3}$ are not multiples of the fundamental frequency and $f_{s}=1000 \mathrm{H}_{Z}$, $f_{1}=33 \mathrm{H}_{Z}, f_{2}=133 \mathrm{H}_{Z}$, and $f_{3}=233 \mathrm{H}_{Z}$. The waveform and spectrum of the signal are shown in Figure 14(a). The parameters of the multiband filter are set as $M=400$, the half bandwidth of passband is $P=8$, and number of passbands is $K=4$. Parameters are set according to the filter design [44] and actual needs. The waveform and spectrum of simulation signal $x(n)$ processed by the multiband filter are shown in Figure 14(b).

From Figure 14, $f_{1}, f_{2}$, and $f_{3}$, as the interference frequency, are filtered out after passing through the multiband filter, while the fundamental frequency $f_{0}$ and its frequency multiplications $2 f_{0}, 3 f_{0}$, and $4 f_{0}$ are well preserved. Therefore, the multiband filter can filter out the frequency components independent of the given 

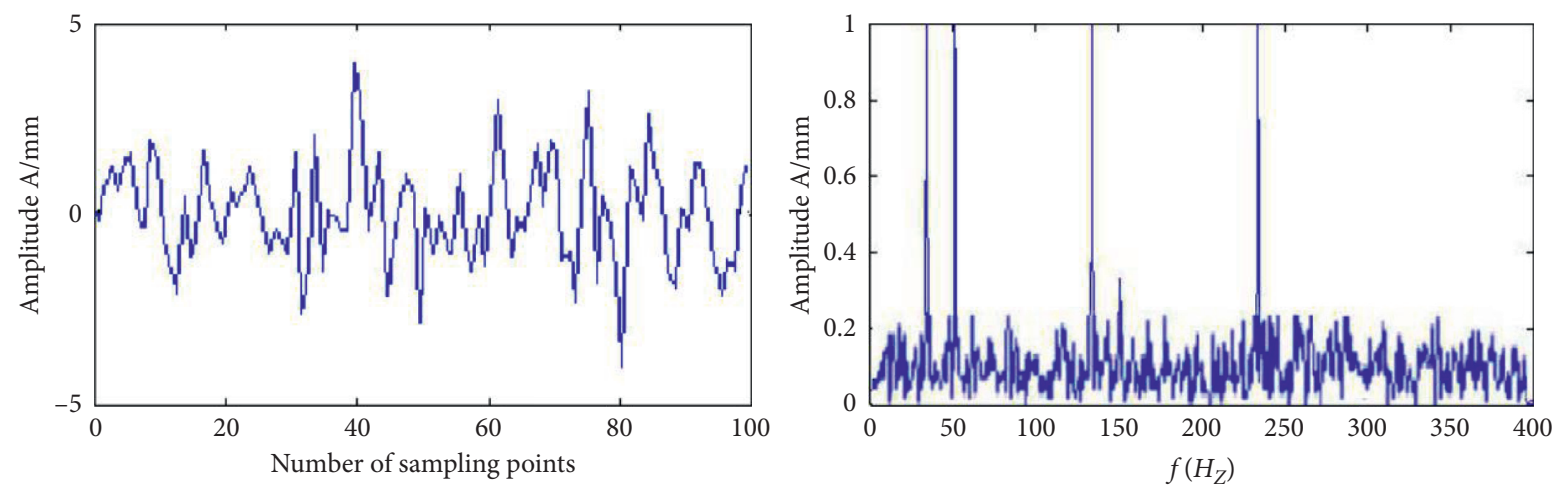

(a)
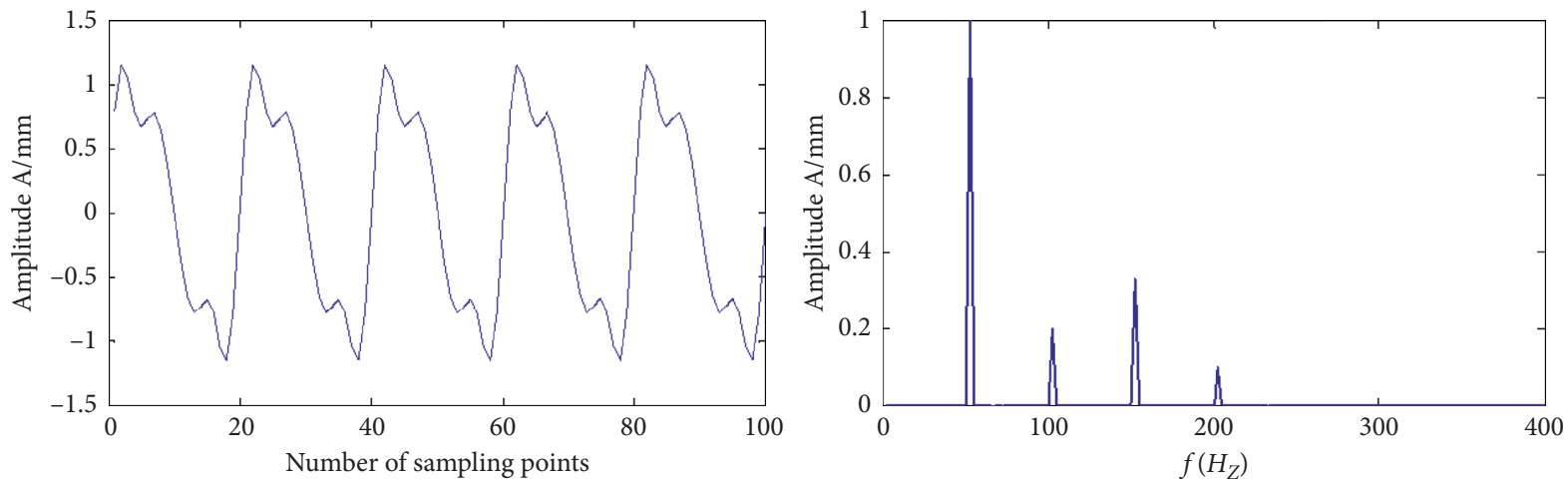

(b)

FigURE 14: Simulation signal analysis results. (a) Original waveform and spectrum and (b) waveform and spectrum processed by the multiband filter.

frequency and retain the frequency components related to a given frequency to perform the same function as the TDA method.

3.5. Experimental Validation. Figure 15(a) shows a group of vibration signals collected from the gear fault simulation test bench. The signal parameters are set as follows: the input shaft speed is $800 \mathrm{rpm}$, the sampling frequency is $5120 \mathrm{H}_{Z}$, the sampling number is 16384 , the number of whole cycles is approximately 40 , and the gear meshing pair is $26 / 64$. The first paragraph signal is taken as reference signal for analysis and processing. The results are shown in Figure 16.

The specific methods are as follows.

(1) For signal $x_{n}$, the fundamental frequency $f_{0}=69.02 \mathrm{H}_{Z}$ of the correction spectrum is calculated by equations (11) and (12), and the fundamental frequency $f_{0}$ of the filter bank to be set is determined.

(2) Considering the sampling frequency range and the frequency components of interest, the number of passband is set as $K=7$.

(3) The frequency response function of the comb filter [45] is obtained by constructing a multiband (comb) filter. The parameter of the multiband filter is set as $M=200$, and the width of the passband is $P=8$.
The signal to be analyzed is filtered through the constructed multiband filter, and the filtering results are shown in Figure 16.

After processing, the waveform signals with serious noise interference, as shown in Figure 15(a), can effectively reflect the time-domain characteristics of the gear operation and can clearly distinguish the impact signals with 26 basically equal intervals, corresponding to the number of teeth on the gears of the input shaft, as shown in Figure 16(a). The amplitude change of each impact presents a process from small to large and then from large to small, illustrating that the gear is not running normally and that there may exist bending or eccentricity problems. From Figure 16(b), the processed signal spectrum is close to the ideal TDA result, making the uncorrelated spectrum components completely suppressed. At the same time, the filter has the advantage of the TDA selectivity analysis signal, as long as the frequency band components of interest are set, and different values for $K$ are selected.

3.6. Feasibility Analysis. The gear signals obtained from the experiment in Section 3.5 were synchronously corrected and then superposed and averaged. The number of segments was 40. The analysis results are shown in Figure 17 and used the multiband filter method and traditional TDA. From Figure 17, the time-domain waveform characteristics of the traditional method are basically consistent with those of the 


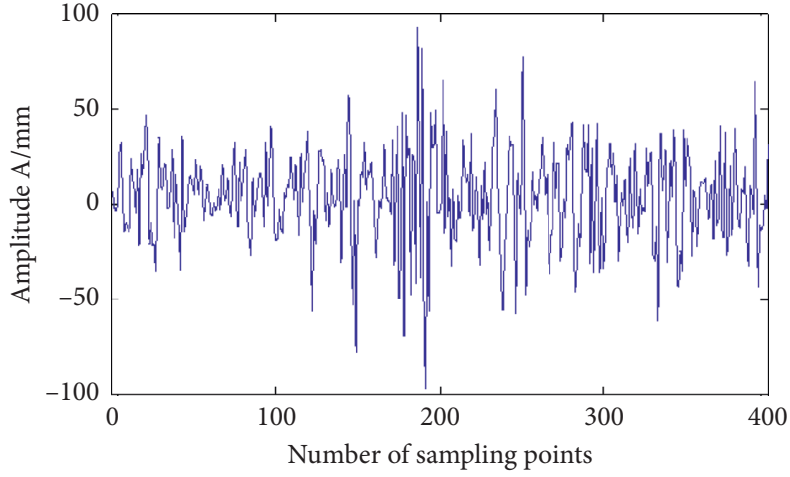

(a)

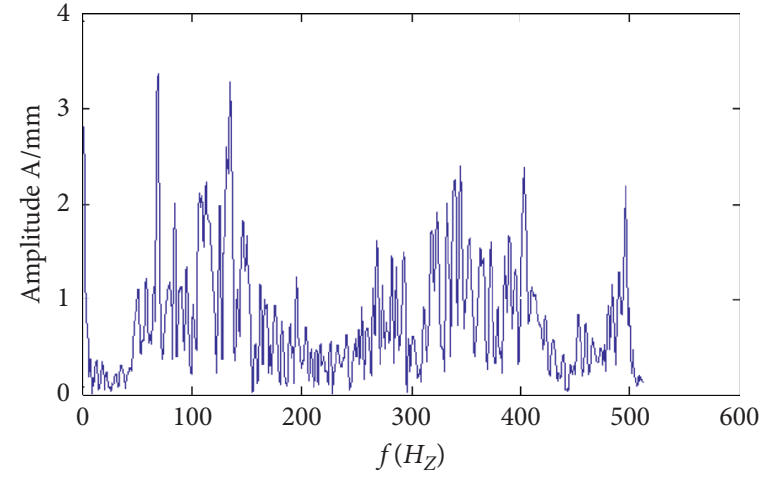

(b)

FIGURE 15: Original signal. (a) Time-domain waveform of the original signal and (b) original signal spectrum.

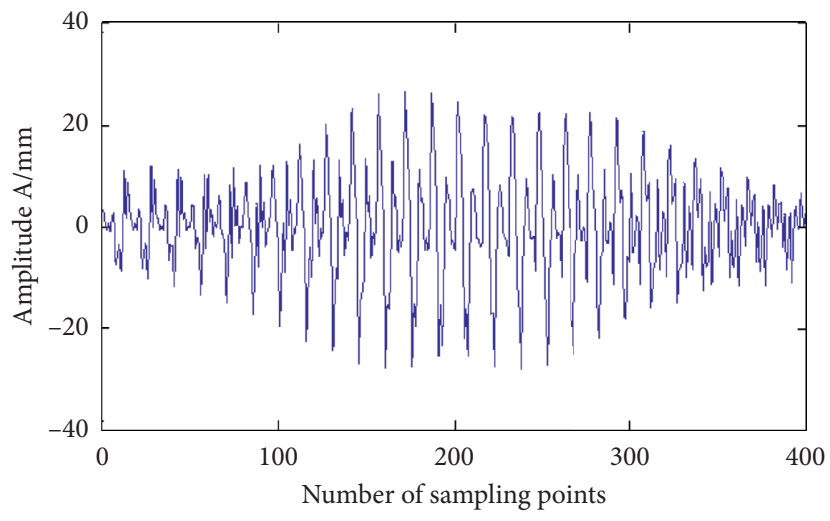

(a)

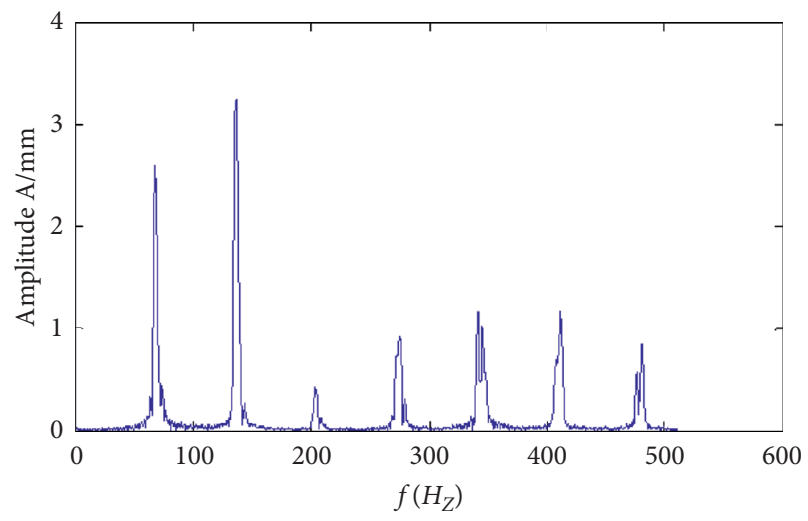

(b)

FIgURE 16: Processed signal. (a) Time-domain waveform of the processed signal and (b) processed signal spectrum.

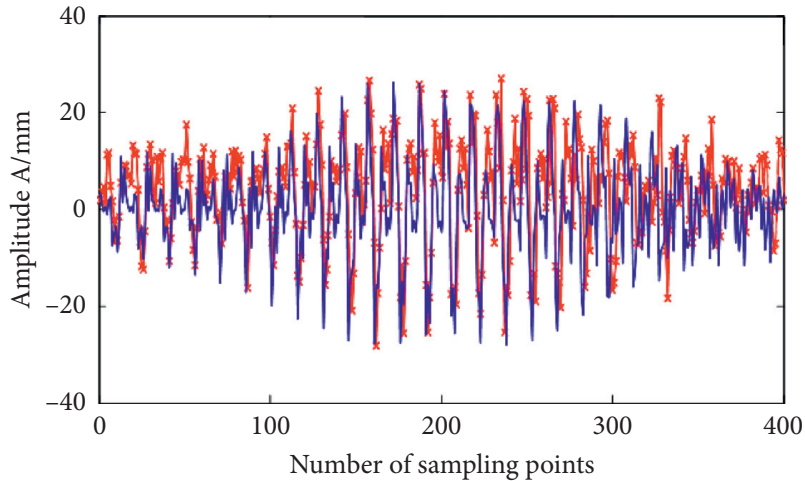

FIGURE 17: Comparison results of two methods of gear signal. The red line is the result of traditional TDA, and the blue line is the result of multiband filter.

multiband filter method, which shows the feasibility of the multiband filter method.

Two methods were used to analyze and compare the bearing colliding fault signals obtained from the tests in Section 2.3. The processed results are shown in Figure 18,

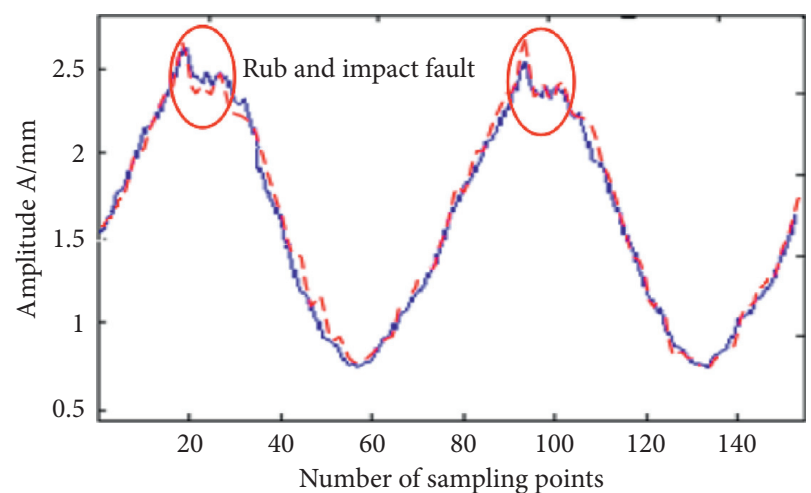

Figure 18: Comparison results of two methods for processing the impact wear signal.

where the dotted line is the result of multiband filtering and the solid line is the TDA processing result. Although the results are not completely consistent, the two methods can reflect the impact wear fault information very well, which further proves the practical feasibility of the multiband filter method, effectively expands the application range of the 
traditional TDA, and greatly simplifies the application conditions of the traditional TDA.

\section{Conclusion}

4.1. Summary. In this paper, the principle of TDA is described, and an improved resampling method, called the segmented resampling method, is proposed to address the synchronicity problem. The effectiveness and practicability of the proposed method are demonstrated by analyzing the simulation signal and the actual signal, respectively. Under the condition of velocity fluctuation, the segmented resampling method broadens the application range of TDA and improves the analysis precision. Then, based on the TDA amplitude-frequency map, this paper proposes a method to realize the TDA function with a multiband filter. The selection, construction, and parameter optimization of the TDA filter are studied, and the feasibility of the method is verified by analyzing and processing the simulated signal and the actual signal. The experimental results of the filter implementation technology show that this method can effectively retain the fundamental frequency and the octave frequency of the periodic signal of interest, filter out the irrelevant interference components, and realize the TDA function.

4.2. Application Comparison. In this section, the calculation complexity is compared. On the premise of reaching a similar result accuracy, the traditional TDA method needs to iterate $N$ times to intercept $N$-segment data, $N$ is 32 in this paper, while the filter implementation method needs to iterate approximately $(N+N 2)$ times according to the construction and filtering process of the multiband filter in the previous paper. Therefore, the calculation requirements of the FIR multiband filter to achieve the TDA function are much larger than that of the traditional TDA method. Next, the practical conditions are compared. Although the improved TDA method improves the synchronization accuracy compared with the traditional TDA, the improved method still needs the acquisition of the keyphasor signals, which is greatly limited by the practical application environment or equipment accuracy. Therefore, it is not difficult to see that realizing the TDA function by the multiband filter overcomes the limitation of the TDA method at the expense of the required amount of operations. Therefore, when the practical situation is not too complex and the conditions of the devices allow, the improved TDA method is preferred because of its simplicity, small calculation amount and tendency to not make mistakes; when the situation is complicated and the practical requirements are high, the filter implementation technology is selected to achieve the TDA function.

\section{Data Availability}

The mechanical vibration signal data used to support the findings of this study are available from the corresponding author upon request.

\section{Conflicts of Interest}

The authors declare that there are no conflicts of interest regarding the publication of this paper.

\section{Acknowledgments}

The work was financially supported by the basic scientific research fees of Zhejiang Ocean University (2019JZ00004), provincial first-class discipline construction project supported by Young and Middle-Aged Discipline Leaders of Zhejiang Province, and Science and Technology Plan Project of Zhoushan Science and Technology Bureau (2018C21014).

\section{References}

[1] A. K. S. Jardine, D. Lin, and D. Banjevic, "A review on machinery diagnostics and prognostics implementing condition-based maintenance," Mechanical Systems and Signal Processing, vol. 20, no. 7, pp. 1483-1510, 2006.

[2] T. Ghosh, D. Bhattacharyya, S. K. Bandyopadhyay, and T.-H. Kim, "A review on different techniques to de-noise a signal," International Journal of Control and Automation, vol. 7, no. 3, pp. 349-358, 2014.

[3] R. Wang, H. Xu, J. Wang, X. Liu, and L. Liu, "Data denosing processing of the operating state of the robotic arm of coal sampling robot," in Proceedings of the International Conference on Intelligent Robotics and Applications, pp. 155-164, Shenyang, China, August 2019.

[4] B. Premanode, J. Vongprasert, and C. Toumazou, "Noise reduction for nonlinear nonstationary time series data using averaging intrinsic mode function," Algorithms, vol. 6, no. 3, pp. 407-429, 2013.

[5] C. R. Trimble, "What is signal averaging," Hewlett-packard Journal, vol. 19, no. 8, pp. 2-7, 1968.

[6] S. Braun, "The extraction of periodic waveforms by time domain averaging," Acustica, vol. 32, no. 2, pp. 69-77, 1975.

[7] P. D. McFadden, "A revised model for the extraction of periodic waveforms by time domain averaging," Mechanical Systems and Signal Processing, vol. 1, no. 1, pp. 83-95, 1987.

[8] D. Luo, "Princple and application of time domain average," Journal of Vibration, Measurement and Diagnosis (China), vol. 19, no. 3, pp. 202-207, 1999.

[9] R. M. Stewart, "Some useful data analysis techniques for gearbox diagnostics," Institute of Sound and Vibration Research, vol. 19, 1977.

[10] P. D. Mcfadden, "Detecting fatigue cracks in gears by amplitude and phase demodulation of the meshing vibration," Journal of Vibration and Acoustics, vol. 108, no. 2, pp. 165170, 1986.

[11] P. D. McFadden, "Examination of a technique for the early detection of failure in gears by signal processing of the time domain average of the meshing vibration," Mechanical Systems and Signal Processing, vol. 1, no. 2, pp. 173-183, 1987.

[12] G. Dalpiaz, "Early detection of fatigue cracks in gears by vibration analysis techniques," Osterreichische Ingenieur- und Architekten-Zeitschrift (OIAZ), vol. 135, pp. 312-317, 1990.

[13] Y. Kim, "Phase-based time domain averaging (PTDA) for fault detection of a gearbox in an industrial robot using vibration signals," Mechanical Systems and Signal Processing, vol. 138, pp. 1-19, 2020.

[14] A. G. A. Rahman, O. Z. Chao, and Z. Ismail, "Effectiveness of Impact-Synchronous Time Averaging in determination of 
dynamic characteristics of a rotor dynamic system," Measurement, vol. 44, no. 1, pp. 34-45, 2011.

[15] N. Ahamed, Y. Pandya, and A. Parey, "Spur gear tooth root crack detection using time synchronous averaging under fluctuating speed," Measurement, vol. 52, pp. 1-11, 2014.

[16] E. J. Cross, K. Worden, and Q. Chen, "Cointegration: a novel approach for the removal of environmental trends in structural health monitoring data," Proceedings of the Royal Society A: Mathematical, Physical and Engineering Sciences, vol. 467, no. 2133, pp. 2712-2732, 2011.

[17] J. H. Stock and M. W. Watson, "Testing for common trends," Journal of the American Statistical Association, vol. 83, no. 404, pp. 1097-1107, 1988.

[18] J. H. Stock and M. W. Watson, "A simple estimator of cointegrating vectors in higher order integrated systems," Econometrica, vol. 61, no. 4, pp. 783-820, 1993.

[19] E. J. Cross, G. Manson, K. Worden, and S. G. Pierce, "Features for damage detection with insensitivity to environmental and operational variations," Proceedings of the Royal Society A: Mathematical, Physical and Engineering Sciences, vol. 468, no. 2148, pp. 4098-4122, 2012.

[20] K. Worden, I. Iakovidis, and E. J. Cross, "New results for the ADF statistic in nonstationary signal analysis with a view towards structural health monitoring," Mechanical Systems and Signal Processing, vol. 146, Article ID 106979, 2021.

[21] F. Combet and L. Gelman, "An automated methodology for performing time synchronous averaging of a gearbox signal without speed sensor," Mechanical Systems and Signal Processing, vol. 21, no. 6, pp. 2590-2606, 2007.

[22] E. Bechhoefer, "A review of time synchronous average algorithms," 2009.

[23] L. Zhao and Y. Guo, "Time domain synchronous averaging based on accurate alignment reference signal," in Proceedings of the 9th International Conference on Modelling, Identification and Control, Kunming, China, July 2017.

[24] S. Braun and B. Seth, "Analysis of repetitive mechanism signatures," Journal of Sound and Vibration, vol. 70, no. 4, pp. 513-526, 1980.

[25] Y. De, "Digital resampling for frequency tracking in engineering signal analysis," British Journal of Pharmacology, vol. 115, no. 4, pp. 672-676, 1997.

[26] J. Zheng and X. L. L. Q. He, "The principle and application of signal time domain average," Signal Processing(China), vol. 2, no. 4 , pp. 236-243, 1986.

[27] T. Sayeda, "The impact of exchange rate on Bangladesh's export: a cointegration approach," Journal of Economics, Management and Trade, vol. 26, no. 7, pp. 22-30, 2020.

[28] D. Chen, "Pairs trading in Chinese commodity futures markets: an adaptive cointegration approach," Accounting \& Finance, vol. 57, no. 5, 2017.

[29] S. Fallahpour, "Pairs trading strategy optimization using the reinforcement learning method: a cointegration approach," Soft Computing, vol. 20, no. 12, 2016.

[30] T. Stoichev, "Multiple regression analysis to assess the contamination with metals and metalloids in surface sediments (Aveiro Lagoon, Portugal)," Marine Pollution Bulletin, vol. 159, Article ID 111470, 2020.

[31] Y. Bin, "Analysis of the key factors of pumping well system efficiency for oil field based on multiple regression," IOP Conference Series: Earth and Environmental Science, vol. 661, no. 1, 2021.

[32] G. Xu and Y. Feng, "Multiple linear regression analysis-source prediction method for predicting mine gas emission," in The 6th Academic Conference of Geology Resource Management and Sustainable Development, Beijing, China, December 2018.

[33] J. Lin, "An integrated time domain averaging scheme for gearbox diagnosis," in IEEE Proceeding: 8th International Conference on Reliability, Maintainability and Safety, IEEE, Chengdu, China, July 2009.

[34] Z. K. Peng and F. L. Chu, "Application of the wavelet transform in machine condition monitoring and fault diagnostics: a review with bibliography," Mechanical Systems and Signal Processing, vol. 18, no. 2, pp. 199-221, 2004.

[35] N. Baydar and A. Ball, "Detection of gear failures via vibration and acoustic signals using wavelet transform," Mechanical Systems and Signal Processing, vol. 17, no. 4, pp. 787-804, 2003.

[36] J. Lin and M. J. Zuo, "Gearbox fault diagnosis using adaptive wavelet filter," Mechanical Systems and Signal Processing, vol. 17, no. 6, pp. 1259-1269, 2003.

[37] N. E. Huang, Z. Shen, S. R. Long et al., "The empirical mode decomposition and the Hilbert spectrum for nonlinear and non-stationary time series analysis," Proceedings of the Royal Society of London. Series A: Mathematical, Physical and Engineering Sciences, vol. 454, no. 1971, pp. 903-995, 1998.

[38] Y. Lei, "A review on empirical mode decomposition in fault diagnosis of rotating machinery," Mechanical Systems and Signal Processing, vol. 35, no. 1-2, pp. 108-126, 2013.

[39] Z. Wu and N. E. Huang, "Ensemble empirical mode decomposition: a noise-assisted data analysis method," Advances in Adaptive Data Analysis, vol. 1, no. 1, pp. 1-41, 2009.

[40] X. Tian and E. Zhang, "A new algorithm for FIR digital filter design based on window function method," Radio Engineering (China), vol. 31, pp. 228-229, 2001.

[41] X. Zhang, "Analysis and simulation of lowpass and bandpass FIR digital filter," Modern Electronics Technique (China), vol. 31, no. 19, pp. 57-59, 2008.

[42] G. Andria, M. Savino, and A. Trotta, "Optimized windows for FIR filter design to perform maximally flat decimation stages in signal conditioning," IEEE Transactions on Instrumentation and Measurement, vol. 41, no. 3, 1991.

[43] D. H. Horrocks and D. R. Bull, "The frequency variation of response errors in fixed and floating point FIR filters," in Proceeding of European Conference on Circuit Theory and Design, Brighton, UK, September 1989.

[44] C. Liu, "Designed principle and program of FIR digital filter with windows function method," Journal of Qiqihar University (China), vol. 3, pp. 35-38, 2001.

[45] H. Yao, "Window function method compare with frequency sampling method of FIR digital filter design," Journal of Anyang Institute of Technology (China), vol. 6, pp. 51-53, 2007. 